\title{
On the Coupling of Boundary Integral and Finite Element Methods
}

\section{By Claes Johnson and J. Claude Nedelec}

Abstract. Let $\Omega^{c}$ be the complementary of a bounded regular domain in $\mathrm{R}^{2}$ of boundary $\Gamma$. We consider the problem

$$
\left\{\begin{array}{l}
\Delta u=f ; \quad \text { in } \Omega^{c}, \\
\left.u\right|_{\Gamma}=u_{0},
\end{array}\right.
$$

where $f$ has its support in a bounded subdomain $\Omega_{1}$ of $\Omega^{c}$. Let $\Gamma_{2}$ be the common boundary of $\Omega_{1}$ and $\Omega_{2}=\Omega^{c}-\Omega_{1}$. We solve the problem (1) by using an equivalent system of equations involving an integral equation on $\Gamma_{2}$ coupled with the equation:

$$
\left\{\begin{aligned}
\Delta u & =f \quad \text { in } \Omega_{1}, \\
\left.u\right|_{\Gamma} & =u_{0}, \\
\left.u\right|_{\Gamma_{2}} & =\lambda .
\end{aligned}\right.
$$

We introduce a finite element approximation of Eq. (2) and of the integral equation and we prove optimal error estimates.

Introduction. The purpose of this note is to analyze a procedure obtained by coupling the boundary integral method (cf. [4] , [5], [7], [8] , [12]) and the usual finite element method. Such coupled procedures have been proposed by e.g. SilvesterHsieh [10] and Zienkiewicz et al. [11] for the numerical solution of problems in unbounded domains. As a typical example let us consider a problem of the form

$$
\left\{\begin{array}{cc}
A u=f & \text { in } \Omega^{c}, \\
u=u_{0} & \text { on } \Gamma,
\end{array}\right.
$$

where $\Omega$ is a bounded domain in the plane with boundary $\Gamma, \Omega^{c}$ is the unbounded complement of $\Omega$, and $A$ is an elliptic differential operator. Let us further assume that $\Omega^{c}$ can be divided into a bounded part $\Omega_{1}$ and an unbounded part $\Omega_{2}$, with common boundary $\Gamma_{2}$ (see Figure 1), so that $f=0$ in $\Omega_{2}$ and $A$ is linear and has constant coefficients in $\Omega_{2}$ while $A$ may be nonlinear or have variable coefficients in the bounded part $\Omega_{1}$. Then the unbounded part $\Omega_{2}$ can be taken into account using an integral equation on the boundary $\Gamma_{2}$, and an approximate solution can be found using a conventional finite element discretization of $\Omega_{1}$ together with a discretization along $\Gamma_{2}$. Below we shall analyze a model problem of this type.

For numerical experiments and references into the engineering literature on this subject, we refer to [11].

Received February 22, 1979; revised December 13, 1979. 1980 Mathematics Subject Classification. Primary 65 N99. 
1. A Model Problem. Let us consider the following exterior Dirichlet problem:

$$
\left\{\begin{aligned}
-\Delta u=f & \text { in } \Omega^{c}, \\
u=0 & \text { on } \Gamma,
\end{aligned}\right.
$$

where $\Omega$ is a bounded domain in $\mathbf{R}^{2}$ with smooth boundary $\Gamma$ and $\Omega^{c}$ is the complement of $\Omega \cup \Gamma$. Let us assume that the support of $f$ is bounded and that $f \in L^{2}\left(\Omega^{c}\right)$. It is known (see e.g. [3] , [6]) that the problem (1.1) admits a unique solution $u \in W^{1}\left(\Omega^{c}\right)$, where

$$
W^{1}\left(\Omega^{c}\right)=\left\{v:\left(1+|x|^{2}\right)^{-1 / 2}\left(1+\log \sqrt{1+|x|^{2}}\right)^{-1} v \in L^{2}\left(\Omega^{c}\right), \nabla v \in\left[L^{2}\left(\Omega^{c}\right)\right]^{2}\right\},
$$

and that this solution has the following asymptotic behavior:

$$
\left\{\begin{aligned}
u(x)=\alpha+0\left(\frac{1}{|x|}\right), & |x| \rightarrow \infty \\
\nabla u(x)=0\left(\frac{1}{|x|^{2}}\right), & |x| \rightarrow \infty
\end{aligned}\right.
$$

where $\alpha$ is a constant.

Let now $\Gamma_{2}$ be a smooth curve dividing $\Omega^{c}$ into an unbounded part $\Omega_{2}$ and a

\begin{tabular}{|c|c|c|}
\hline$(1.3 \mathrm{a})$ & $-\Delta u_{1}=f$ & in $\Omega_{1}$, \\
\hline$(1.3 b)$ & $-\Delta u_{2}=f=0$ & in $\Omega_{2}$ \\
\hline$(1.3 c)$ & $u_{1}=u_{2}$ & on $\Gamma_{2}$, \\
\hline$(1.3 \mathrm{~d})$ & $\frac{\partial u_{1}}{\partial n}=\frac{\partial u_{2}}{\partial n}=\lambda$ & on $\Gamma_{2}$ \\
\hline$(1.3 \mathrm{e})$ & $u_{1}=0$ & on $\Gamma$, \\
\hline
\end{tabular}
bounded part $\Omega_{1}$ containing the support of $f$ (see Figure 1). Then (1.1) can alternatively be formulated as follows:

where $u_{i}=u \mid \Omega_{i}, i=1,2$, and $\partial / \partial n$ denotes the outward normal derivative to $\Gamma_{2}=$ $\partial \Omega_{2}$ (see Figure 1). The equations (1.3a) and (1.3b) signify a decomposition into two problems in the separate domains $\Omega_{1}$ and $\Omega_{2}$, while $(1.3 \mathrm{c})$ and $(1.3 \mathrm{~d})$ reflect the appropriate coupling of these two problems.

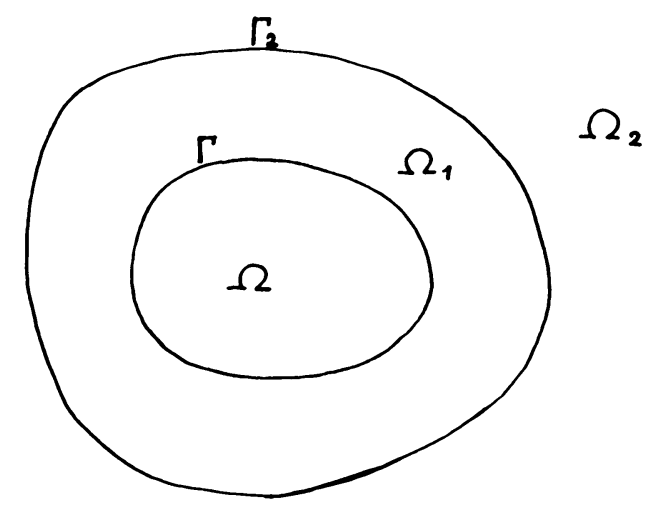

FIGURE 1 
2. A Variational Formulation of the Model Problem. Let us now give a variational formulation of (1.2). Since $-\Delta u=f$ in $\Omega_{1}$, we find, using Green's formula, that

$$
a(u, v)+\langle v, \lambda=(f, v) \quad \forall v \in W
$$

where

$$
\begin{gathered}
\lambda=\frac{\partial u}{\partial n} \mid \Gamma_{2}, \quad a(u, v)=\int_{\Omega_{1}} \nabla u \cdot \nabla v d x, \\
\left\langle v, \lambda=\int_{\Gamma_{2}} v \lambda d s, \quad(f, v)=\int_{\Omega_{1}} f v d x,\right. \\
W=\left\{v \in H^{1}\left(\Omega_{1}\right): v=0 \text { on } \Gamma\right\} .
\end{gathered}
$$

Moreover, since $-\Delta u=0$ in $\Omega_{2}$, we find, using Green's formula and (1.2), that (cf.

[6]),

$$
\begin{aligned}
\frac{1}{2} u(x) & =\int_{\Gamma_{2}} u(y) G_{n}(x, y) d s_{y}-\int_{\Gamma_{2}} \lambda(y) G(x, y) d s_{y}+\alpha, \quad x \in \Gamma_{2}, \\
u(x) & =\int_{\Gamma_{2}} u(y) G_{n}(x, y) d s_{y}-\int_{\Gamma_{2}} \lambda(y) G(x, y) d s_{y}+\alpha, \quad x \in \Omega_{2},
\end{aligned}
$$

where

$$
G(x, y)=\frac{1}{2 \pi} \log |x-y|, \quad x \neq y,
$$

is the Green's function associated with the two-dimensional Laplacian and

$$
G_{n}(x, y)=\frac{\partial}{\partial n_{y}} G(x, y), \quad x \neq y, y \in \Gamma_{2},
$$

with $n_{y}$ being the outward unit normal to $\Gamma_{2}$ at $y \in \Gamma_{2}$. Let us observe that (1.2) together with (2.3) imply that $\int_{\Gamma_{2}} \lambda d s=0$, since otherwise $u(x)$ would behave like $c \log |x|, c \neq 0$, as $|x| \rightarrow \infty$, thus contradicting (1.2).

Now, formally multiplying (2.2) by the function $\mu(x)$ satisfying $\int_{\Gamma_{2}} \mu d s=0$, and integrating over $\Gamma_{2}$, we find that

$$
b(\lambda, \mu)-\frac{1}{2}\langle u, \mu\rangle+\left\langle G_{n} u, \mu\right\rangle=0
$$

where

$$
\begin{aligned}
& b(\lambda, \mu)=-\int_{\Gamma_{2}} \int_{\Gamma_{2}} \lambda(y) \mu(x) G(x, y) d s_{x} d s_{y}, \\
& G_{n} u(x)=\int_{\Gamma_{2}} u(x) G_{n}(x, y) d s_{y} .
\end{aligned}
$$

We recall (see [6]) that $b$ is a continuous bilinear form on $H^{-1 / 2}\left(\Gamma_{2}\right) \times H^{-1 / 2}\left(\Gamma_{2}\right)$. Moreover, $b$ is $H$-elliptic with

$$
H=\left\{\mu \in H^{-1 / 2}\left(\Gamma_{2}\right):\langle 1, \mu\rangle=0\right\},
$$

i.e., there exists a positive constant $\beta$ such that

$$
b(\mu, \mu) \geqslant \beta|\mu|_{-1 / 2}^{2}, \quad \mu \in H .
$$


Here $\langle\cdot, \cdot\rangle$ denotes the duality between $H^{1 / 2}\left(\Gamma_{2}\right)$ and $H^{-1 / 2}\left(\Gamma_{2}\right)$ and we use the notation

$$
\mid \cdot I_{s}=\|\cdot\|_{H^{s}\left(\Gamma_{2}\right)} \cdot
$$

Recalling (2.1) and (2.4), we are thus led to the following variational formulation of (1.2): Find $(u, \lambda) \in W \times H$ such that

$$
\begin{cases}a(u, v)+\langle v, \lambda\rangle=(f, v) & \forall v \in W \\ 2 b(\lambda, \mu)-\langle u, \mu\rangle+2\left\langle G_{n} u, \mu\right\rangle=0 & \forall \mu \in H\end{cases}
$$

Let us now analyze this problem. First, recalling the trace theorem:

$$
|\gamma v|_{s-1 / 2} \leqslant C_{s}\|v\|_{s} \quad \forall v \in H^{s}\left(\Omega_{1}\right)
$$

where $s>1 / 2, \gamma v=\left.v\right|_{\Gamma_{2}}$ and $\|\cdot\|_{s}=\|\cdot\|_{H^{s}\left(\Omega_{1}\right)}$, it follows that $\langle\cdot, \cdot\rangle$ is a continuous bilinear form on $W^{\prime} \times H$. Further, since $v=0$ on $\Gamma$ if $v \in W$, it follows that $a(\cdot, \cdot)$ is $W$-elliptic, i.e., there is a positive constant $\beta^{\prime}$ such that

$$
a(v, v) \geqslant \beta^{\prime}\|v\|_{1}^{2} \quad \forall v \in W .
$$

Moreover, since

$$
G_{n}(x, y)=-\frac{n_{y} \cdot(x-y)}{|x-y|^{2}}, \quad x, y \in \Gamma_{2},
$$

and $\Gamma$ is smooth, it follows that $G_{n}$ is pseudo-homogeneous of degree zero and thus (see [9]) the integral operator $G_{n}$ defined by (2.5) is smoothing. More precisely, one has

$$
\left|G_{n} v\right|_{s+1} \leqslant C_{s}|v|_{s} \quad \forall v \in H^{s}\left(\Gamma_{2}\right) .
$$

In order to analyze (2.7), it is convenient to introduce the following simplified problem obtained by omitting the term $\left\langle G_{n} u, \mu\right\rangle$ : Given $\hat{g}=\left(g_{1}, g_{2}, g_{3}\right)$ find $(w, \theta) \in$ $W \times H$ such that

$$
\begin{cases}a(w, v)+\langle v, \theta\rangle=\left(g_{1}, v\right)+\left\langle v, g_{2}\right\rangle & \forall v \in W, \\ 2 b(\theta, \mu)-\langle w, \mu\rangle=\left\langle g_{3}, \mu\right\rangle & \forall \mu \in H .\end{cases}
$$

We shall see that due to (2.11) the full problem (2.7) is a compact perturbation of the simplified problem (2.12).

Let us now formulate (2.7) and (2.12) as operator equations. To this end we introduce the continuous bilinear forms

$$
A, B, K: V \times V \rightarrow \mathbf{R},
$$

where $V=W \times H$, and the corresponding continuous linear mappings

$$
A, B, K: V \rightarrow V^{\prime},
$$


defined by

$$
\begin{aligned}
& B(\hat{u}, \hat{v}) \equiv[B \hat{u}, \hat{v}] \equiv a(u, v)+\langle v, \lambda-\langle u, \mu\rangle+2 b(\lambda, \mu), \\
& K(\hat{u}, \hat{v}) \equiv[K \hat{u}, \hat{v}]=\left\langle G_{n} u, \mu\right\rangle \quad \forall \hat{u}=(u, \lambda), \hat{v}=(v, \mu) \in V, \\
& A=B+K,
\end{aligned}
$$

where $[\cdot, \cdot]$ denotes the duality between $V$ and $V^{\prime}$, the dual of $V$. Then (2.12) can be formulated

$$
B \hat{u}=\hat{g}, \quad \hat{u}=(u, \lambda),
$$

i.e.,

$$
B(\hat{u}, \hat{v})=[\hat{g}, \hat{v}] \quad \forall \hat{v}=(v, \lambda) \in V
$$

and (2.7) is equivalent to

$$
A \hat{u}=\hat{f}
$$

i.e.,

$$
A(\hat{u}, \hat{v})=[\hat{f}, \hat{v}] \quad \forall \hat{v} \in V
$$

with $\hat{f}=(f, 0,0)$.

Let us note that the bilinear form $B(\cdot, \cdot)$ is $V$-elliptic; by $(2.6)$ and (2.9) we have that

$$
\begin{aligned}
B(\hat{v}, \hat{v}) & =a(v, v)+2 b(\mu, \mu) \\
& \geqslant\left.\beta^{\prime}\right|_{i v}\left\|_{1}^{2}+2 \beta|\mu|_{-1 / 2}^{2} \geqslant \beta^{\prime \prime} \mid i \hat{v}\right\|_{V}^{2} \quad \forall \hat{v} \in V,
\end{aligned}
$$

where $\beta^{\prime \prime}=\min \left(\beta^{\prime}, 2 \beta\right)$ and $\|\cdot\|_{V}$ denotes the norm in $V$, i.e.,

$$
\|\hat{v}\|_{V}^{2}=\left(\|v\|_{1}^{2}+|\mu|_{-1 / 2}^{2}\right)^{1 / 2} \text {. }
$$

Lemma 1. The mapping $B: V \longrightarrow V^{\prime}$ is an isomorphism. Moreover, for $k \geqslant 0$ the mapping

$$
B^{-1}: H^{k-1}\left(\Omega_{1}\right) \times H^{k-1 / 2}\left(\Gamma_{2}\right) \times H^{k+1 / 2}\left(\Gamma_{2}\right) \rightarrow H^{k+1}\left(\Omega_{1}\right) \times H^{k-1 / 2}\left(\Gamma_{2}\right),
$$

defined by $B \hat{u}=\hat{g}$ is continuous.

Proof. The first statement of the lemma follows directly from the $V$-ellipticity (2.17). The regularity result is proved in the Appendix below.

Let us now return to the original problem $A \hat{u}=\hat{f}$. Since $A=B+K$, this problem can be written after applying $B^{-1}$ :

$$
\left(I+B^{-1} K\right) \hat{u}=B^{-1} \hat{f}
$$

where $I: V \rightarrow V$ is the identity mapping. Now, recalling (2.8) and (2.11), it follows that $K: V \rightarrow\{0\} \times\{0\} \times H^{3 / 2}\left(\Gamma_{2}\right)$, is continuous. Therefore, using Lemma 1 with $k=1$, we see that $B^{-1} K: V \rightarrow H^{2}\left(\Omega_{1}\right) \times H^{1 / 2}\left(\Gamma_{2}\right)$ is continuous. Since $H^{2}\left(\Omega_{1}\right) \times$ $H^{1 / 2}\left(\Gamma_{2}\right)$ is compactly embedded in $V$, it follows that $B^{-1} K: V \rightarrow V$ is compact and 
hence (2.18) is an equation of the Fredholm second kind. Thus, to prove existence of a solution to $(2.18)$ or the equivalent original problem $(2.15)$, it is sufficient to prove uniqueness. With this observation it is easy to prove

Lemma 2. The mapping $A: V \rightarrow V^{\prime}$ is an isomorphism. Moreover, for $k \geqslant 0$ the mapping

$$
A^{-1}: H^{k-1}\left(\Omega_{1}\right) \times H^{k-1 / 2}\left(\Gamma_{2}\right) \times H^{k+1 / 2}\left(\Gamma_{2}\right) \rightarrow H^{k+1}\left(\Omega_{1}\right) \times H^{k-1 / 2}\left(\Gamma_{2}\right)
$$

is continuous.

Proof. To prove uniqueness of the solution of the equation $A \hat{u}=\hat{f}$, let us assume that $\hat{w}=(w, \theta) \in V$ and $A \hat{w}=0$, i.e.,

$$
\left\{\begin{array}{l}
a(w, v)+\langle v, \theta\rangle=0 \quad \forall v \in W, \\
2 b(\theta, \mu)-\langle w, \mu\rangle+2\left\langle G_{n} w, \mu\right\rangle=0 \quad \forall \mu \in H .
\end{array}\right.
$$

From (2.19a) it follows that

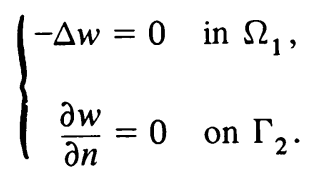

Let now $\widetilde{w} \in W^{1}\left(\Omega_{2}\right)$ be the harmonic extension of $w$ to $\Omega_{2}$, i.e.,

$$
\left\{\begin{aligned}
-\Delta \widetilde{w} & =0 & & \text { in } \Omega_{2}, \\
\widetilde{w} & =\gamma w & & \text { on } \Gamma .
\end{aligned}\right.
$$

Then, by an argument similar to that leading to (2.4), it follows that

$$
2 b(\tilde{\theta}, \mu)-\langle w, \mu\rangle+2\left\langle G_{n} w, \mu\right\rangle=0, \quad \mu \in H,
$$

where $\tilde{\theta}=\partial \tilde{w} /\left.\partial n\right|_{\Gamma_{2}} \in H$. Combining (2.19b) and (2.21) we find that $b(\tilde{\theta}-\theta, \mu)=$ $0 \forall \mu \in H$, and thus (2.6) shows that $\theta=\tilde{\theta}$. But this means that if $w$ is extended to $\Omega^{c}$ by putting $w=\widetilde{w}$ in $\Omega_{2}$, then $\Delta w=0$ in $\Omega^{c}, w \in W^{1}\left(\Omega^{c}\right)$, and $w=0$ on $\Gamma$ so that $w \equiv 0$ and the uniqueness follows. Thus, for any $\hat{g} \in V^{\prime}$, the equation $A \hat{w}=\hat{g}$ has a unique solution and the continuity of $A^{-1}: V^{\prime} \rightarrow V$ follows from the closed graph theorem. This proves the first statement of the lemma.

To prove the regularity result, we use induction on $k$. Thus assume that the statement holds for $k=m-1$. Let us consider the equation $A \hat{w}=\hat{g}$, where $\hat{g} \in H^{m-1}\left(\Omega_{1}\right) \times H^{m-1 / 2}\left(\Gamma_{2}\right) \times H^{m+1 / 2}\left(\Gamma_{2}\right)$. By the induction hypothesis, we then have $\hat{w} \in H^{m}\left(\Omega_{1}\right) \times H^{m-3 / 2}\left(\Gamma_{2}\right)$ so that by (2.11) $K \hat{w} \in\{0\} \times\{0\} \times H^{m+1 / 2}\left(\Gamma_{2}\right)$. But the equation $A \hat{w}=\hat{g}$ can be written

$$
B \hat{w}=\hat{g}-K \hat{w},
$$

and thus by Lemma 1 we conclude that $\hat{w} \in H^{m+1}\left(\Omega_{1}\right) \times H^{m-1 / 2}\left(\Gamma_{2}\right) \times$ $H^{m+1 / 2}\left(\Gamma_{2}\right)$. Therefore $A^{-1}$ maps $H^{m-1}\left(\Omega_{1}\right) \times H^{m-1 / 2}\left(\Gamma_{2}\right) \times H^{m+1 / 2}\left(\Gamma_{2}\right)$ into $H^{m+1}\left(\Omega_{1}\right) \times H^{m-1 / 2}\left(\Gamma_{2}\right)$ and the continuity of the mapping follows from the closed graph theorem. This completes the induction step and thus the proof of the lemma. 
We shall also need the corresponding result for the adjoints $B^{*}, A^{*}: V \rightarrow V^{\prime}$ defined by

$$
\begin{aligned}
& {\left[A^{*} \hat{v}, \hat{w}\right]=[A \hat{w}, \hat{v}],} \\
& {\left[B^{*} \hat{v}, \hat{w}\right]=[B \hat{w}, \hat{v}] \quad \forall \hat{v}, \hat{w} \in V .}
\end{aligned}
$$

Lemma 3. The mappings $B^{*}, A^{*}: V \rightarrow V^{\prime}$ are isomorphisms and, for $k \geqslant 0$,

$$
B^{*-1}, A^{*-1}: H^{k-1}\left(\Omega_{1}\right) \times H^{k-1 / 2}\left(\Gamma_{2}\right) \times H^{k+1 / 2}\left(\Gamma_{2}\right) \rightarrow H^{k+1}\left(\Omega_{1}\right) \times H^{k-1 / 2}\left(\Gamma_{2}\right)
$$

are continuous.

The proof is parallel to the proofs of Lemmas 1 and 2 .

Remark. As pointed out by the referee, if the outer boundary $\Gamma_{2}$ is a circle, then one can solve the equation (2.3) in $\lambda$ explicitly. More precisely, in this case (2.3) takes the form

$$
-\frac{1}{\pi} \int_{0}^{2 \pi} \tilde{\lambda}(\theta) \log \left(2\left|\sin \frac{\theta-\eta}{2}\right|\right) R d \theta=\gamma(\eta),
$$

where $\tilde{\lambda}(\theta)=\lambda(R \cos \theta, R \sin \theta)$ and $\gamma$ is determined by $u$. This integral equation has the explicit solution

$$
\tilde{\lambda}(\theta)=\frac{1}{2 \pi R} \int_{0}^{2 \pi} \frac{d \gamma}{d \eta} \operatorname{cotan}\left(\frac{\eta-\theta}{2}\right) d \eta-\frac{1}{2 \pi R\left(\log R^{2}\right)} \int_{0}^{2 \pi} \gamma d \eta,
$$

which makes it possible to eliminate $\lambda$ from (2.7) and thus obtain an equation involving only $u$. To see if such a procedure is advantageous from a numerical point of view requires further investigation.

3. The Coupled Procedure. Error Estimates. Let us now consider a finite element method based on the variational formulation (2.7). Let $W_{h} \subset W$ and $H_{h} \subset H$ be finite-dimensional spaces depending on the positive parameter $h$ and set $V_{h}=$ $w_{h} \times H_{h}$. Let $A_{h}(\cdot, \cdot)$ be a bilinear form approximating $A(\cdot, \cdot)$ and consider the following discrete problem: Find $\hat{u}_{h}=\left(u_{h}, \lambda_{h}\right) \in V_{h}$ such that

$$
A_{h}\left(\hat{u}_{h}, \hat{v}\right)=(f, v) \quad \forall \hat{v} \in V_{h} .
$$

We shall assume that the spaces $W_{h}$ and $H_{h}$ satisfy the following approximation hypothesis: For any positive $\epsilon$, there exists a constant $C$ such that

$$
\begin{array}{ll}
\operatorname{Inf}_{v \in W_{h}}\|w-v\|_{1} \leqslant C h^{s}\|w\|_{s+1+\epsilon}, & 0 \leqslant s \leqslant k, \\
\operatorname{Inf}_{\mu \in H_{h}}|\theta-\mu|_{-1 / 2} \leqslant C h^{s}|\theta|_{s-1 / 2}, & 0 \leqslant s \leqslant k,
\end{array}
$$

where $k$ is a positive integer. This will correspond to using piecewise polynomials of degree $k$ for $W_{h}$ and degree $k-1$ for $H_{h}$ (cf. Example 1 below). Note that the functions in $H_{h}$ may be chosen to be discontinuous while the functions in $W_{h}$ will have to be continuous. Furthermore, we shall assume that there is a constant $C$ such that

$$
\left|A(\hat{v}, \hat{w})-A_{h}(\hat{v}, \hat{w})\right| \leqslant C h^{k}\|\hat{v}\|_{V}\|\hat{w}\|_{V} \quad \forall \hat{v}, \hat{w} \in V_{h} .
$$


In Example 1 below we shall in detail consider a finite element method satisfying (3.2) and (3.3) with $k=1$.

We shall now prove that for $h$ small enough the problem (3.1) admits a unique solution $\hat{u}_{h}$ and then estimate the error $\hat{u}-\hat{u}_{h}$. The crucial result is then the following:

LEMmA 4. There is a positive constant $c$ such that for $h$ small enough

$$
\sup _{\hat{v} \in V_{h}, \hat{v} \neq 0} \frac{A_{h}(\hat{w}, \hat{v})}{\|\hat{v}\|_{V}} \geqslant c \mid\|\hat{w}\|_{V} \quad \forall \hat{w} \in V_{h} .
$$

Proof. Given $\hat{w} \in V_{h}$ there exists by Lemma $3 \hat{\psi}=(\psi, H) \in V$ such that

$$
A(\hat{v}, \hat{\psi})=(\hat{w}, \hat{v})_{V}, \quad \hat{v} \in V,
$$

where $(\cdot, \cdot)_{V}$ denotes the scalar product in $V$, and

$$
\|\hat{\psi}\|_{V} \leqslant C\|\hat{w}\|_{V}
$$

In fact, $\hat{\psi}=A^{*-1} J \hat{w}$, where $J: V \rightarrow V^{\prime}$, is the canonical mapping defined by [J $\left.\hat{w}, \hat{v}\right]$ $=(\hat{w}, \hat{v})_{V}, \forall \hat{v}, \hat{w} \in V$. Furthermore, again by Lemma 3 , there exists $\hat{\psi}_{h}=\left(\psi_{h}, H_{h}\right) \in$ $V_{h}$ such that

$$
B\left(\hat{v}, \hat{\psi}-\hat{\psi}_{h}\right)=0 \quad \forall \hat{v} \in V_{h},
$$

and

$$
\left\|\hat{\psi}_{h}\right\|_{V} \leqslant C\|\hat{\psi}\|_{V}
$$

Now, using (3.7) and (3.5) with $v=w$, we find that

$$
\begin{aligned}
A\left(\hat{w}, \hat{\psi}_{h}\right) & =B\left(\hat{w}, \hat{\psi}_{h}\right)+K\left(\hat{w}, \hat{\psi}_{h}\right) \\
& =B(\hat{w}, \hat{\psi})+K\left(\hat{w}, \hat{\psi}_{h}\right)=A(\hat{w}, \hat{\psi})+K\left(\hat{w}, \hat{\psi}_{h}-\hat{\psi}\right) \\
& =\|\hat{w}\|_{V}^{2}+\left\langle G_{n} \hat{w}, H_{h}-H\right\rangle \\
& \geqslant\|\hat{w}\|_{V}^{2}-\left|G_{n} w\right|_{3 / 2}\left|H-H_{h}\right|_{-3 / 2} \\
& \geqslant\|\hat{w}\|_{V}^{2}-\|\hat{w}\|_{V}\left|H-H_{h}\right|_{-3 / 2},
\end{aligned}
$$

since by (2.11) and (2.8),

$$
\left|G_{n} w\right|_{3 / 2} \leqslant C|w|_{1 / 2} \leqslant C\|w\|_{1} \leqslant C\|\hat{w}\|_{V}
$$

In order to estimate $\left|H-H_{h}\right|_{-3 / 2}$, we shall use the usual duality argument: Given $v \in H^{3 / 2}\left(\Gamma_{2}\right)$ let $\hat{\varphi}=B^{-1} \hat{v}$ where $\hat{v}=(0,0, v)$, i.e.,

$$
B(\hat{\varphi}, \hat{v})=\langle v, \mu\rangle \quad \forall \hat{v}=(v, \mu) \in V .
$$

By Lemma 2 we then have

$$
\|\hat{\varphi}\|_{H^{2}\left(\Omega_{1}\right) \times H^{1 / 2}\left(\Gamma_{2}\right)} \leqslant C|v|_{3 / 2} .
$$


Hence, taking $\hat{v}=\hat{\psi}-\hat{\psi}_{h}$ in (3.10) and using (3.7), we find that for any $\hat{\varphi}_{h} \in V_{h}$,

$$
\begin{aligned}
\left\langle v, H-H_{h}\right\rangle & =B\left(\hat{\varphi}, \hat{\psi}-\hat{\psi}_{h}\right)=B\left(\hat{\varphi}-\hat{\varphi}_{h}, \hat{\psi}-\hat{\psi}_{h}\right) \\
& \leqslant C\left\|\hat{\varphi}-\hat{\varphi}_{h}\right\|_{V}\left\|\hat{\psi}-\hat{\psi}_{h}\right\|_{V} \leqslant C\left\|\hat{\varphi}-\hat{\varphi}_{h}\right\|_{V}\|\hat{w}\|_{V},
\end{aligned}
$$

where the last inequality follows from (3.6) and (3.8). Thus, using (3.2) with $s=1-\epsilon$ together with (3.11), it follows that

$$
\left\langle v, H-H_{h}\right\rangle \leqslant C h^{1-\epsilon}|v|_{3 / 2}\|\hat{w}\|_{V}, \quad v \in H^{3 / 2}\left(\Gamma_{2}\right),
$$

which proves that

$$
\left|H-H_{h}\right|_{-3 / 2} \leqslant C h^{1-\epsilon}\|\hat{w}\|_{V},
$$

where $0<\epsilon<1$. Returning to (3.9) we thus have

$$
A\left(\hat{w}, \hat{\psi}_{h}\right) \geqslant\|\hat{w}\|_{V}^{2}-C h^{1-\epsilon}\|\hat{w}\|_{V}^{2}=\left(1-C h^{1-\epsilon}\right)\|\hat{w}\|_{V}^{2} .
$$

Finally, recalling (3.3), we conclude that

$$
A_{h}\left(\hat{w}, \hat{\psi}_{h}\right)=A\left(\hat{w}, \hat{\psi}_{h}\right)+A_{h}\left(\hat{w}, \hat{\psi}_{h}\right)-A\left(\hat{w}, \hat{\psi}_{h}\right) \geqslant\left(1-C h^{1-\epsilon}\right)\|\hat{w}\|_{V}^{2} .
$$

Since $\left\|\hat{\psi}_{h}\right\|_{V} \leqslant C\|\hat{w}\|_{V}$, this proves that (3.4) holds for $h$ sufficiently small and the proof is complete.

We can now prove

THEOREM 1. For $h$ sufficiently small the discrete problem (3.1) admits a unique solution $\hat{u}_{h} \in V_{h}$ and we have the following error estimate:

$$
\left\|\hat{u}-\hat{u}_{h}\right\|_{V} \leqslant C h^{k}\|u\|_{k+1+\epsilon} .
$$

Proof. Uniqueness, and hence existence, of a solution of (3.1) for $h$ sufficiently small follows directly from Lemma 4. Furthermore, using (2.16), (3.1), (3.3) and Lemma 4, we see that for any $\hat{v}_{h} \in V_{h}$,

$$
\begin{aligned}
\left\|\hat{u}_{h}-\hat{v}_{h}\right\|_{V} & \leqslant C \sup _{\hat{v} \in V_{h}} \frac{A_{h}\left(\hat{u}_{h}-\hat{v}_{h}, \hat{v}\right)}{\|\hat{v}\|_{V}} \\
& =C \sup _{\hat{v} \in V_{h}} \frac{A\left(\hat{u}-\hat{v}_{h}, \hat{v}\right)+A\left(\hat{v}_{h}, \hat{v}\right)-A_{h}\left(\hat{v}_{h}, \hat{v}\right)}{\|\hat{v}\|_{V}} \\
& \leqslant C\left\|\hat{u}-\hat{v}_{h}\right\|_{V}+C h^{k}\left\|\hat{v}_{h}\right\|_{V} .
\end{aligned}
$$

Thus, choosing $\hat{v}_{h}$ according to (3.2), we find that

$$
\begin{aligned}
\left\|\hat{u}-\hat{u}_{h}\right\|_{V} & \leqslant\left\|\hat{u}-\hat{v}_{h}\right\|_{V}+\left\|\hat{u}_{h}-\hat{v}_{h}\right\|_{V} \\
& \leqslant C h^{k}\left(\|u\|_{k+1+\epsilon}+|\lambda|_{k-1 / 2}\right) .
\end{aligned}
$$

Finally, by the trace theorem (2.8), we have

$$
|\lambda|_{k-1 / 2} \leqslant C\|u\|_{k+1}
$$

and the proof is complete. 
Let us now exhibit a natural finite element method satisfying (3.2) and (3.3) with $k=1$.

Example 1. Let $\Gamma_{2}$ be chosen so that $\bar{\Omega} \cup \Omega_{1}$ is convex and let $\Omega_{1}^{h} \subset \Omega_{1}$ be a polygonal domain approximating $\Omega_{1}$ according to Figure 2 . Let $\Gamma^{h}$ and $\Gamma_{2}^{h}$ be the corresponding polygonal approximations of $\Gamma$ and $\Gamma_{2}$ so that $\partial \Omega_{1}^{h}=\Gamma^{h} \cup \Gamma_{2}^{h}$. Let $S_{h}=\{S\}$ be the sides of $\Gamma_{2}^{h}$, let $h$ be the maximal length of the sides $S \in S_{h}$, and define

$$
\widetilde{H}_{h}=\left\{\mu \in L^{2}\left(\Gamma_{h}\right):\left.\mu\right|_{S} \text { is constant, } S \in S_{h},\langle 1, \mu\rangle_{h}=0\right\},
$$

where $\langle v, \mu\rangle_{h}=\int_{\Gamma^{h}} v \mu d s$. Further, let $T_{h}=\{T\}$ be a regular* triangulation of $\Omega_{1}^{h}$ with maximal sidelength at most $h$ and define

$$
\widetilde{W}_{h}=\left\{v \in H^{1}\left(\Omega_{1}^{h}\right):\left.v\right|_{T} \text { is linear, } T \in T_{h}, v=0 \text { on } \Gamma^{h}\right\} .
$$

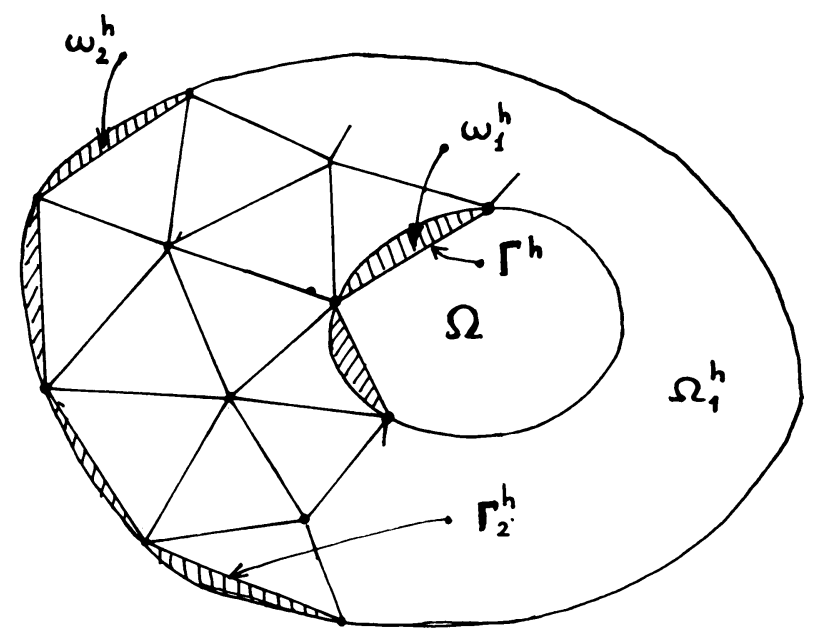

FIGURE 2

In order to formulate a discrete analogue of (2.7), using the spaces $\widetilde{W}_{h}$ and $\widetilde{H}_{h}$ and replacing boundary integrals along $\Gamma$ by integrals along the polygonal boundary $\Gamma_{2}^{h}$, we have to rewrite the term $\left\langle G_{n} u, \mu\right\rangle$. To this end we note that taking $u \equiv 1$ in (2.2) shows that

$$
\int_{\Gamma_{2}} G_{n}(x, y) d s_{y}=-1 / 2, \quad x \in \Gamma_{2} .
$$

Hence recalling (2.10), we have

$$
\begin{aligned}
\left\langle G_{n} u, \mu\right\rangle & =\int_{\Gamma_{2}} \int_{\Gamma_{2}} G_{n}(x, y)[u(y)-u(x)] \mu(x) d s_{y} d s_{x}-1 / 2\langle u, \mu\rangle \\
& =-\frac{1}{2 \pi} \int_{\Gamma_{2}} \int_{\Gamma_{2}} \frac{n_{y} \cdot(x-y)}{|x-y|^{2}}(u(y)-u(x)) \mu(x) d s_{y} d s_{x}-1 / 2\langle u, \mu\rangle \\
& \equiv d(u, \mu)-1 / 2\langle u, \mu\rangle,
\end{aligned}
$$

*All angles of the triangles $T \in T_{h}$ are bounded below uniformly in $h$. 
with the obvious definition of $d(u, \mu)$. As a discrete analogue of the form $d(\cdot, \cdot)$, we now introduce the form $d_{h}(\cdot, \cdot)$ defined by

$$
\tilde{d}_{h}(w, \mu)=-\frac{1}{2 \pi} \int_{\Gamma_{2}^{h}} \int_{\Gamma_{2}^{h}} \frac{n_{y h} \cdot(x-y)}{|x-y|^{2}}(w(y)-w(x)) \mu(x) d s_{y} d s_{x},
$$

where $n_{h y}$ is linear on each $S \in S_{h}$ and $n_{h y}$ interpolates the normal $n_{y}$ at the vertices of $\Gamma_{2}^{h}$. We note that, since functions in $W_{h}$ are Lipschitz continuous, the form $\tilde{d}_{h}(\cdot, \cdot)$ is well defined on $W_{h} \times H_{h}$.

We can now formulate the following discrete analogue of (2.7): Find $\left(\tilde{u}_{h}, \tilde{\lambda}_{h}\right) \in$ $\widetilde{W}_{h} \times \widetilde{H}_{h}$ such that

$$
\left\{\begin{array}{l}
a_{h}\left(\tilde{u}_{h}, v\right)+\left\langle v, \tilde{\lambda}_{h} \tilde{\nu}_{h}=(f, v)_{h} \quad \forall v \in \tilde{W}_{h},\right. \\
2 \tilde{b}_{h}\left(\tilde{\lambda}_{h}, \mu\right)-2\left\langle\tilde{u}_{h}, \mu \tilde{h}_{h}+2 \tilde{d}_{h}\left(\tilde{u}_{h}, \mu\right)=0 \quad \forall \mu \in \tilde{H}_{h},\right.
\end{array}\right.
$$

where

$$
\begin{aligned}
a_{h}(u, v) & =\int_{\Omega_{1}^{h}} \nabla u \cdot \nabla v d x, \\
\left\langle v, \nu_{h}^{\sim}\right. & =\int_{\Gamma_{2}^{h}} v \lambda d s, \\
\tilde{b}_{h}(\lambda, \mu) & =-\int_{\Gamma_{2}^{h}} \int_{\Gamma_{2}^{h}} \lambda(y) \mu(x) G(x, y) d s_{y} d s_{x} .
\end{aligned}
$$

The problem (3.13) will lead to a nonsymmetric linear system of equations where we have one unknown per node in the triangulation $T_{h}$ of $\Omega_{1}^{h}$ and one unknown per side of the polygonal boundary $\Gamma_{2}^{h}$. The coefficients corresponding to the forms $a_{h}(\cdot, \cdot)$ and $\langle\cdot, \cdot\rangle_{h}$ are easy to compute. Algorithms for computing the coefficients corresponding to the forms $\tilde{b}_{h}(\cdot, \cdot)$ and $\tilde{d}_{h}$ can be found in [2].

Let us now show that the problem (3.13) can be put into the form (3.1) with assumptions (3.2) and (3.3) fulfilled. First, in order to convert the spaces $\widetilde{W}_{h}$ and $\widetilde{H}_{h}$ into subspaces $W_{h} \subset W$ and $H_{h} \subset H$, we introduce the mapping $\psi: \Gamma_{2}^{h} \rightarrow \Gamma_{2}$, where $\psi(x)$ is the point on $\Gamma_{2}$ closest to the point $x \in \Gamma_{2}^{h}$. For $h$ small enough $\psi$ is clearly a bijection. Now, using the mapping $\psi^{-1}$ to transform integrals along $\Gamma_{2}^{h}$ to integrals along $\Gamma_{2}$, we have

$$
\int_{\Gamma_{2}^{h}} \mu d s=\int_{\Gamma_{2}} \mu \circ \psi^{-1} J\left(\psi^{-1}\right) d s,
$$

where $J\left(\psi^{-1}\right)=\left|\partial \psi^{-1} / \partial s\right|$, and $\partial / \partial s$ denotes differentiation in the tangential direction to $\Gamma_{2}$. We now define

$$
H_{h}=\left\{\mu: \mu=J\left(\psi^{-1}\right) \tilde{\mu} \circ \psi^{-1}, \tilde{\mu} \in \widetilde{H}_{h}\right\} .
$$

Note that if $\mu=J\left(\psi^{-1}\right) \tilde{\mu} \circ \psi^{-1} \in H_{h}$ with $\tilde{\mu} \in \tilde{H}_{h}$, then

$$
0=\int_{\Gamma_{2}^{h}} \tilde{\mu} d s=\int_{\Gamma_{2}} \tilde{\mu} \circ \psi^{-1} J\left(\psi^{-1}\right) d s=\int_{\Gamma_{2}} \mu d s,
$$

so that $H_{h} \subset H$. 
Furthermore, we extend each function $\widetilde{w} \in \widetilde{W}_{h}$ defined in $\Omega_{1}^{h}$ to a function $w$ defined in $\Omega_{1}$ by setting $w=0$ in the "skin" $\omega_{1}^{h}$ with boundary $\Gamma \cup \Gamma^{h}$ and finally by setting $w(y)=w(x)$ for $y$ on the line segment between $x \in \Gamma_{2}^{h}$ and $\psi(x)$ thus defining $w$ in the "skin" $\omega_{2}^{h}$ with boundary $\Gamma_{2} \cup \Gamma_{2}^{h}$ (see Figure 2). We denote by $W_{h}$ the set of functions obtained in this way.

By changing integrations from $\Gamma_{2}^{h}$ to $\Gamma_{2}$ and using the definitions of $H_{h}$ and $W_{h}$, the problem (3.13) can now be formulated as follows: Find $\left(u_{h}, \lambda_{h}\right) \in W_{h} \times H_{h}$ such that

$$
\left\{\begin{array}{l}
a_{h}\left(u_{h}, v\right)+\left\langle v, \lambda_{h}\right\rangle=(f, v) \quad \forall v \in W_{h}, \\
2 b_{h}\left(\lambda_{h}, \mu\right)-2\left\langle u_{h}, \mu\right\rangle+2 d_{h}\left(u_{h}, \mu\right)=0 \quad \forall \mu \in H_{h},
\end{array}\right.
$$

where

$$
\begin{gathered}
b_{h}(\lambda, \mu)=-\int_{\Gamma_{2}} \int_{\Gamma_{2}} \lambda(y) \mu(x) \log \left|\left(\psi^{-1}(y)-\psi^{-1}(x)\right)\right| d s_{y} d s_{x} \\
d_{h}(u, \mu)=-\frac{1}{2 \pi} \int_{\Gamma_{2}} \int_{\Gamma_{2}} \frac{n_{y h} \cdot\left(\psi^{-1}(x)-\psi^{-1}(y)\right)}{\left|\psi^{-1}(x)-\psi^{-1}(y)\right|^{2}}(u(y)-u(x)) \mu(x) \\
\times J\left(\psi^{-1}(y)\right) d s_{y} d s_{x} .
\end{gathered}
$$

Let us now check that the assumptions (3.2) and (3.3) are satisfied with $k=1$ and

$$
A_{h}(v, w)=a_{h}(v, w)+\langle w, \lambda\rangle-2\langle v, \mu\rangle+2 b_{h}(\lambda, \mu)+2 d_{h}(v, \mu) .
$$

To prove (3.2a) let $w \in H^{2}\left(\Omega_{1}\right)$ be given and let $w_{h} \in \widetilde{W}$ interpolate $w$ at the nodes of $T_{h}$. Then, by well-known interpolation theory (see [1]),

$$
\left\|w-w_{h}\right\|_{H^{1}\left(\Omega_{1}^{h}\right)} \leqslant C h\|w\|_{2} .
$$

By Sobolev's embedding theorem we have, for any $\epsilon>0$,

$$
\|\nabla w\|_{L^{\infty}\left(\Omega_{1}\right)} \leqslant C\|w\|_{2+\epsilon}
$$

and hence also

$$
\left\|\nabla w_{h}\right\|_{L^{\infty}\left(\Omega_{1}\right)} \leqslant C\|w\|_{2+\epsilon} .
$$

Since the area of $\Omega_{1}-\Omega_{1}^{h}$ is of the order $O\left(h^{2}\right)$, this proves that

$$
\left\|w-w_{h}\right\|_{H^{1}\left(\Omega_{1} \backslash \Omega_{1}^{h}\right)} \leqslant C h\|w\|_{2+\epsilon},
$$

and thus (3.2a) follows. For a proof of (3.2b) we refer to [6] .

It remains to prove (3.3). First, since by the construction of $W_{h}$,

we find that

$$
\left\|w_{h}\right\|_{H^{1}\left(\omega_{2}^{h}\right)} \leqslant C h^{1 / 2}\left\|w_{h}\right\|_{1}, \quad w_{h} \in w_{h},
$$

$$
\left|a\left(w_{h}, v_{h}\right)-a_{h}\left(w_{h}, v_{h}\right)\right|=\int_{\omega_{2}^{h}} \nabla w_{h} \cdot \nabla v_{h} d x \leqslant C h\left\|w_{h}\right\|_{1}\left\|v_{h}\right\|_{1} .
$$


Next, since (see [5])

$$
\left|\frac{\left|\psi^{-1}(x)-\psi^{-1}(y)\right|}{|x-y|}-1\right| \leqslant C h^{2}
$$

it follows easily that

$$
\left|b(v, \mu)-b_{h}(v, \mu)\right| \leqslant C h^{2}|v|_{0}|\mu|_{0} .
$$

By the "inverse estimate" (see [6])

$$
|\mu|_{0} \leqslant C h^{-1 / 2}|\mu|_{-1 / 2}, \quad \mu \in H_{h},
$$

we thus have

$$
\left|b(v, \mu)-b_{h}(v, \mu)\right| \leqslant C h|v|_{-1 / 2}|\mu|_{-1 / 2} .
$$

Finally, using (3.16) we find that, for $\epsilon>0$,

$$
\begin{aligned}
& \left|d(w, \mu)-d_{h}(w, \mu)\right| \leqslant C h^{2} \int_{\Gamma_{h}^{2}} \int_{\Gamma_{h}^{2}} \frac{|w(y)-w(x)|}{|x-y|} \mu(x) d s_{x} d s_{y} \\
& \quad \leqslant C h^{2}\left(\int_{\Gamma_{2}^{h}} \int_{\Gamma_{2}^{h}} \frac{|\mu(x)|^{2}}{|x-y|^{1-\epsilon}} d s_{x} d s_{y}\right)^{1 / 2}\left(\int_{\Gamma_{2}^{h}} \int_{\Gamma_{2}^{h}} \frac{|w(y)-w(x)|^{2}}{|x-y|^{1+\epsilon}} d s_{x} d s_{y}\right)^{1 / 2} \\
& \quad \equiv C h^{2} F_{1} F_{2},
\end{aligned}
$$

where we have used Cauchy's inequality and $F_{1}$ and $F_{2}$ are defined in the obvious way. Integrating with respect to $y$ in the factor $F_{1}$, we get $F_{1} \leqslant|\mu|_{0}$. Further (see [5]), for $0<\epsilon<2, F_{2} \leqslant C|w|_{\epsilon / 2}$, and therefore, taking $\epsilon=1$, we have again, using (3.18),

$$
\left|d(w, \mu)-d_{h}(w, \mu)\right| \leqslant C h^{2}|w|_{1 / 2}|\mu|_{0} \leqslant C h^{3 / 2}\|w\|_{1}|\mu|_{-1 / 2} .
$$

Combining (3.15), (3.19), and (3.20), it follows that (3.3) is valid with $k=1$ and thus the verification is complete.

We can also construct analogous methods satisfying (3.2) and (3.3) for $k>1$ using polynomials of degree $k$ for $\widetilde{W}_{h}$ and degree $k-1$ for $\widetilde{H}_{h}$. In such a case the domain $\Omega_{1}$ will be approximated by a domain $\Omega_{1}^{h}$ with piecewise polynomial boundary $\Gamma^{h} \cup \Gamma_{2}^{h}$ of degree $k$ approximating $\Gamma \cup \Gamma_{2}$. In the triangulation of $\Omega_{1}^{h}$, it is then natural to use isoparametric elements of degree $k$ with one curved edge along $\Gamma^{h} \cup \Gamma_{2}^{h}$ (cf. [1]).

4. Error Estimates in Weaker Norms. Let us now, using a duality argument, prove an error estimate in a norm weaker than the norm in $V$. We shall then make the following assumption: For any $\epsilon>0$ there exists a constant $C$ such that if $\hat{w}_{h} \in V_{h}$ interpolates $\hat{w} \in X$, then

$$
\left|A\left(\hat{u}_{h}, \hat{w}_{h}\right)-A_{h}\left(\hat{u}_{h}, \hat{w}_{h}\right)\right| \leqslant C h^{k+1-\epsilon}\|\hat{u}\|_{X}\|\hat{w}\|_{X},
$$


where $X=H^{2}\left(\Omega_{1}\right) \times H^{1 / 2}\left(\Gamma_{2}\right)$. We have

THEOREM 2. For any $\epsilon>0$ there exists a constant $C$ such that if $\hat{u} \in H^{k+1}\left(\Omega_{1}\right)$ $\times H^{k-1 / 2}\left(\Gamma_{2}\right), k \geqslant 1$, then

$$
\left\|\hat{u}-\hat{u}_{h}\right\|_{L^{2}\left(\Omega_{1}\right) \times H^{-3 / 2}\left(\Gamma_{2}\right)} \leqslant C h^{k+1-\epsilon}\|u\|_{k+1+\epsilon} .
$$

Proof. Given $\hat{\varphi} \in L^{2}\left(\Omega_{1}\right) \times H^{3 / 2}\left(\Gamma_{2}\right)$, let $\hat{\psi} \in V$ satisfy

$$
A(\hat{v}, \hat{\psi})=[\hat{v}, \hat{\varphi}] \quad \forall \hat{v} \in V .
$$

By Lemma 3 we then have

$$
\|\hat{\psi}\|_{X} \leqslant C\|\hat{\varphi}\|_{L^{2}\left(\Omega_{1}\right) \times H^{3 / 2}\left(\Gamma_{2}\right)}
$$

Taking $\hat{v}=\hat{u}-\hat{u}_{h}$ in (4.2), recalling (2.16), (3.1), and (3.2), and using (4.1), letting $\hat{\psi}_{h} \in V_{h}$ interpolate $\hat{\psi}$, we find that

$$
\begin{aligned}
{\left[\hat{u}-\hat{u}_{h}, \hat{\varphi}\right] } & =A\left(\hat{u}, \hat{u}_{h}, \hat{\psi}\right) \\
& =A\left(\hat{u}-\hat{u}_{h}, \hat{\psi}-\hat{\psi}_{h}\right)+A_{h}\left(\hat{u}_{h}, \hat{\psi}_{h}\right)-A\left(\hat{u}_{h}, \hat{\psi}_{h}\right) \\
& \leqslant\left\|\hat{u}-\hat{u}_{h}\right\|_{V}\left\|\hat{\psi}-\hat{\psi}_{h}\right\|_{V}+C h^{k+1-\epsilon}\|\hat{u}\|_{X}\|\hat{\psi}\|_{X} \\
& \leqslant C\left(h^{1-\epsilon}\left\|\hat{u}-\hat{u}_{h}\right\|_{V}+h^{k+1-\epsilon}\|\hat{u}\|_{X}\right)\|\hat{\varphi}\|_{L^{2}\left(\Omega_{1}\right) \times H^{3 / 2}\left(\Gamma_{2}\right)} .
\end{aligned}
$$

Together with Theorem 1 , this proves that

$$
\left[\hat{u}-\hat{u}_{h}, \hat{\varphi}\right] \leqslant C h^{k+1-\epsilon}|| \hat{\varphi} \|_{L^{2}\left(\Omega_{1}\right) \times H^{3 / 2}\left(\Gamma_{2}\right)} \forall \hat{\varphi} \in L^{2}\left(\Omega_{1}\right) \times H^{3 / 2}\left(\Gamma_{2}\right),
$$

and the lemma follows.

Remark. It is easy to see that the method of Example 1 satisfies (4.1) with $k=1$. Furthermore, if we define $u_{h}(x)$ for $x \in \Omega_{2}$ by

$$
u_{h}(x)=\frac{1}{2} \int_{\Gamma_{2}^{h}}\left(\tilde{u}_{h}(y)-\tilde{u}_{h}(x)\right) \frac{n_{y h} \cdot(x-y)}{|x-y|^{2}} d s_{y}-\frac{1}{2} \int_{\Gamma_{2}^{h}} \tilde{\lambda}_{h}(y) G(x, y) d s_{y},
$$

then, for all $x \in \Omega_{2}$ with $\operatorname{dist}\left(x, \Gamma_{2}\right) \geqslant \delta>0$ and $h$ sufficiently small, we have $\left|u(x)-u_{h}(x)\right| \leqslant C_{x} h^{2-\epsilon}$, where the constant $C_{x}$ depends on dist( $\left.x, \Gamma\right)$ (cf. [7]).

5. A Symmetrized Procedure. The solution of the original problem (1.1) can be characterized as the solution of the minimization problem

$$
\min _{w \in W^{1}\left(\Omega^{c}\right)}\left\{\frac{1}{2} \int_{\Omega^{c}}|\nabla w|^{2} d x-\int_{\Omega^{c}} f w d x\right\} .
$$

Since $f=0$ in $\Omega_{2}$, this problem can be formulated in the following way:

$$
\min _{w \in V}\left\{\frac{1}{2} \int_{\Omega_{1}}|\nabla w|^{2} d x+\frac{1}{2} \int_{\Omega_{2}}|\nabla \widetilde{w}|^{2} d x-\int_{\Omega_{1}} f w d x\right\}
$$

where $\widetilde{w} \in W^{1}\left(\Omega_{2}\right)$ is the harmonic extension of $w$ according to (2.20). Since $\widetilde{w}$ is harmonic in $\Omega_{2}$, we have by Green's formula $\int_{\Omega_{2}}|\nabla \tilde{w}|^{2}=\langle w, D w\rangle$, where $D: H^{1 / 2}\left(\Gamma_{2}\right) \longrightarrow H$ is the continuous operator defined by $D w=\partial \widetilde{w} /\left.\partial n\right|_{\Gamma_{2}}$. Thus (5.1) 
can be formulated as follows:

$$
\min _{w \in W}\left\{\frac{1}{2} \int_{\Omega_{1}}|\nabla w|^{2} d x+\frac{1}{2}\langle w, D w\rangle-\int_{\Omega_{1}} f w\right\}
$$

The solution $u \in W$ of this problem is characterized by the relation

$$
a(u, v)+\frac{1}{2}\{\langle v, D u\rangle+\langle u, D v\rangle\}=(f, v) \quad \forall v \in W .
$$

Recalling the formulation (2.7), we have that (2.7b) can equivalently be written $\lambda=D u$ and thus $(2.7 \mathrm{c})$ becomes

$$
a(u, v)+\langle v, D u\rangle=(f, v) \quad \forall v \in W .
$$

By Green's formula we have

$$
\langle v, D u\rangle=\langle u, D v\rangle, \quad v, u \in W,
$$

and hence (5.2) and (5.3) are equivalent. Thus the problem (5.3) obtained from (2.7), eliminating the variable $\lambda$, is in fact symmetric. Let us check if the discretized problem (3.13) of Example 1 has the same feature. Introducing the mapping $D_{h}: W_{h} \rightarrow H_{h}$ defined by

$$
b_{h}\left(D_{h} w_{h}, \mu\right)-\left\langle w_{h}, \mu\right\rangle+d_{h}\left(w_{h}, \mu\right)=0 \quad \forall \mu \in H_{h},
$$

the problem (3.13) can be written: Find $u_{h} \in W_{h}$ such that

$$
a_{h}\left(u_{h}, v\right)+\left\langle v, D_{h} u_{h}\right\rangle=(f, v) \quad \forall v \in W_{h} .
$$

Now, in contrast to (5.4), we have in general $\left\langle v, D_{h} w\right\rangle \neq\left\langle w, D_{h} v\right\rangle$, and thus (5.5) will in general lead to a nonsymmetric system of equations.

In order to obtain a symmetric problem, which will facilitate the incorporation of the coupled procedure into existing finite element codes, it is natural to consider the following variant of (5.5): Find $u_{h} \in W_{h}$ such that

$$
a_{h}\left(u_{h}, v\right)+1 / 2\left\{\left\langle v, D_{h} u_{h}\right\rangle+\left\langle u_{h}, D_{h} v\right\rangle\right\}=(f, v) \quad \forall v \in W_{h},
$$

or, equivalently, the minimization problem:

$$
\min _{w \in W_{h}}\left\{1 / 2 a_{h}(w, w)+1 / 2\left\langle w, D_{h} w\right\rangle-(f, w)\right\}
$$

The problem (5.6) can also be formulated: Find $\hat{u}_{h} \in V_{h}$ such that

$$
\widetilde{A}_{h}\left(\hat{u}_{h}, \hat{v}\right)=\left(f, v ; \quad \forall \hat{v} \in V_{h},\right.
$$

where

$$
\widetilde{A}_{h}(\hat{w}, \hat{v})=A_{h}(\hat{w}, \hat{v})+1 / 2\left\{\left\langle w, D_{h} v\right\rangle-\left\langle v, D_{h} w\right\rangle\right\} .
$$

We shall prove the following lemma which extends the result of Section 3 to the symmetrized problems (5.6), (5.7). 
Lemma 3. There exists a constant $C$ such that for $\hat{v}, \hat{w} \in \hat{V}_{h}$,

$$
\left|A_{h}(\hat{w}, \hat{v})-\widetilde{A}_{h}(\hat{w}, \hat{v})\right| \leqslant C h\|\hat{v}\|_{V}\|\hat{w}\|_{V} .
$$

Proof. By the definition of $D_{h} v$, it follows easily that

$$
\begin{aligned}
\delta_{h} & \equiv 2\left|A_{h}(\hat{w}, \hat{v})-\widetilde{A}_{h}(\hat{w}, \hat{v})\right|=\left|\left\langle w, D_{h} v\right\rangle-\left\langle v, D_{h} w\right\rangle\right| \\
& =\left|d_{h}\left(w, D_{h} v\right)-d_{h}\left(v, D_{h} w\right)\right| .
\end{aligned}
$$

On the other hand, by (5.4) and the definition of $D$, we have

$$
d(w, D v)=d(v, D w), \quad v, w \in W
$$

and thus

$$
\begin{aligned}
\delta_{h} \leqslant & \left|d_{h}\left(w, D_{h} v\right)-d\left(w, D_{h} v\right)\right|+\left|d_{h}\left(v, D_{h} w\right)-d\left(v, D_{h} w\right)\right| \\
& +\left|d\left(w, D_{h} v-D v\right)-d\left(v, D_{h} w-D w\right)\right| \\
& \equiv \delta_{h 1}+\delta_{h 2}+\delta_{h 3},
\end{aligned}
$$

with obvious notation. The first two terms can be estimated using (3.20). Rewriting the remaining term using (3.12), we get

$$
\begin{aligned}
\delta_{h 3} & =\left|\left\langle G_{n} w, D_{h} v-D v\right\rangle-\left\langle G_{n} v, D_{h} w-D w\right\rangle\right| \\
& \leqslant C\left[\left|G_{n} w\right|_{3 / 2}\left|\left(D-D_{h}\right) v\right|_{-3 / 2}+\left|G_{n} v\right|_{3 / 2}\left|\left(D-D_{h}\right) v\right|_{-3 / 2}\right] .
\end{aligned}
$$

Now, by a standard duality argument (see e.g. [6]), we have that

$$
\left|\left(D-D_{h}\right) v\right|_{-3 / 2} \leqslant C h|v|_{1 / 2} \leqslant C h\|v\|_{1} .
$$

Moreover,

$$
\left|G_{n} v\right|_{3 / 2} \leqslant C|v|_{1 / 2} \leqslant C\|v\|_{1}
$$

and thus $\delta_{h 3} \leqslant C h\|v\|_{1}\|w\|_{1}$, which completes the proof.

Remark. The results of Section 4 can also easily be extended to the problem (5.7).

6. Appendix. We shall here briefly indicate a proof of the regularity result of Lemma 1. We want to prove that, for $k \geqslant 0$,

$$
\|w\|_{k+1}+|\theta|_{k-1 / 2} \leqslant C\left(\left\|g_{1}\right\|_{k-1}+\left|g_{2}\right|_{k-1 / 2}+\left|g_{3}\right|_{k+1 / 2}\right)
$$

if $(w, \theta) \in W \times H$ satisfies (2.12). To this end let us first reformulate (2.12b): We have (cf. [4]) that $\theta \in H$ satisfies (2.12b) if and only if

$$
\theta=\left[\frac{\partial \varphi}{\partial n}\right]=\left.\frac{\partial \varphi}{\partial n}\right|_{\text {int } \Gamma_{2}}-\left.\frac{\partial \varphi}{\partial n}\right|_{\text {ext } \Gamma_{2}},
$$

where $\varphi \in W^{1}\left(\mathbf{R}^{2}\right)$ satisfies

$$
\begin{aligned}
-\Delta \varphi & =0 & & \text { in } \mathbf{R}^{2} \backslash \Gamma_{2}, \\
\varphi & =1 / 2\left(w+g_{3}\right)+c & & \text { on } \Gamma_{2} ;
\end{aligned}
$$


here $[\partial \varphi / \partial n]$ denotes the jump in the normal derivative across $\Gamma_{2}$ and $c$ is a suitable constant. By Green's formula we get from (6.2) and (6.3a) that

$$
D(\varphi, \psi)-\langle\psi, \theta\rangle=0 \quad \forall \psi \in W^{1}\left(\mathbf{R}^{2}\right)
$$

where

$$
D(\varphi, \psi)=\int_{\mathbf{R}^{2}} \nabla \varphi \cdot \nabla \psi d x
$$

Recalling (2.12a), we also have

$$
a(w, v)+\langle v, \theta\rangle=\left(g_{1}, v\right)+\left\langle v, g_{2}\right\rangle \quad \forall v \in W .
$$

Now, to prove (6.1) for $k=0$, we take $v=w$ in (6.5), $\psi=\varphi$ in (6.4) multiply by two and add. Using (6.3b) we then obtain

$$
a(w, w)+2 D(\varphi, \varphi)=\left(g_{1}, w\right)+\left\langle w, g_{2}\right\rangle+\left\langle g_{3}, \theta\right\rangle,
$$

and thus (cf. [6])

$$
\|w\|_{1}^{2}+\|\varphi\|_{W^{1}\left(\mathbf{R}^{2}\right)}^{2} \leqslant C\left[\left(\left\|g_{1}\right\|_{-1}+\left|g_{2}\right|_{-1 / 2}\right)\|w\|_{1}+|\theta|_{-1 / 2}\left|g_{3}\right|_{1 / 2}\right] .
$$

This proves (6.1) in the case $k=0$. For $k \geqslant 1$ we parallel the argument in [8] using the $W \times W^{1}\left(\mathbf{R}^{2}\right)$-ellipticity of the form

$$
\check{D}(\check{w}, \check{v})=a(w, v)+2 D(\varphi, \Psi), \quad \check{w}=(w, \varphi), \quad v=(\check{v}, \Psi) \in W \times W_{0}^{1}\left(\mathbf{R}^{2}\right) .
$$

Institutionen för Informationsbehandling

Chalmers Technological Institute

Fack 40220 Göteborg, Sweden

Centre de Mathématiques Appliquées

Ecole Polytechnique

91128 Palaiseau Cedex, France

1. P. G. CIARLET, The Finite Element Method for Elliptic Problems, North-Holland, Amsterdam, 1978.

2. M. DJAOUA, Equations Intégrales pour un Problème Singulier dans le Plan, These 3é cycle, Paris, 1977.

3. J. GIROIRE, Rapport Interne à l'Ecole Polytechnique, Centre de Mathématiques Appliquées, Palaiseau, 1976.

4. D. GREENSPAN \& P. WERNER, "A numerical method for the exterior Dirichlet problem for the reduced wave equation," Arch. Rational Mech. Anal., v. 23, 1966/67, pp. 288-316.

5. G. C. HSIAO \& W. L. WENDLAND, "A finite element method for some integral equations of the first kind," J. Math. Anal. Appl., v. 58, 1977, pp. 449-481.

6. M. N. LE ROUX, "Méthode d'éléments finis pour la résolution numérique de problèmes extérieurs en dimension 2," R.A.I.R.O. Anal. Numér., v. 11, 1977, pp. 27-60.

7. J. C. NEDELEC, Cours de l'Ecole d'Eté d'Analyse Numérique, C.E.A., I.R.I.A., E.P.F., 1977.

8. J. C. NEDELEC \& J. PLANCHARD, "Une méthode variationelle d'éléments finis pour la résolution numérique d'un problème extérieur dans $\mathrm{R}^{3}$," R.A.I.R.O., v. 7, 1973, pp. 105-129.

9. R. SEELEY, Cours du C.I.M.E. de 1968, ed. Cremonese, Roma, 1969.

10. P. SILVESTER \& M. S. HSIEH, "Finite element solution of 2-dimensional exterior field problems," Proc. Inst. Electr. Engrs., v. 118, 1971, pp. 1743-1746.

11. O. C. ZIENKIEWICZ, D. W. KELLY \& P. BETTESS, "The coupling of the finite element method and boundary solution procedures," Internat. J. Numer. Methods Engrg., v. 11, 1977, pp. $355-375$.

12. W. L. WENDLAND, Elliptic Systems in the Plane, Pitman, New York, 1979. 